\title{
Multimedia Transmissions over a MANET Testbed: Problems and Issues
}

\author{
Elis Kulla*, Makoto Ikeda ${ }^{\dagger}$, Tetsuya Oda*, Leonard Barolli ${ }^{\dagger}$, Fatos Xhafa ${ }^{\ddagger}$ and Makoto Takizawa ${ }^{\S}$ \\ * Graduate School of Engineering \\ Fukuoka Institute of Technology (FIT) \\ 3-30-1 Wajiro-Higashi, Higashi-Ku, Fukuoka 811-0295, Japan \\ Email: bd10002@bene.fit.ac.jp, oda.tetsuya.fit@gmail.com \\ $\dagger$ Department of Information and Communication Engineering \\ Fukuoka Institute of Technology (FIT) \\ 3-30-1 Wajiro-Higashi, Higashi-Ku, Fukuoka 811-0295, Japan \\ Email: makoto.ikd@gmail.com, barolli@fit.ac.jp \\ ¥ Technical University of Catalonia \\ Department of Languages and Informatics Systems \\ C/Jordi Girona 1-3, 08034 Barcelona, Spain \\ E-mail:fatos@lsi.upc.edu \\ $\S$ Department of Computer and Information Science, Seikei University \\ 3-3-1 Kichijoji-kitamachi, Musashino, Tokyo, Japan \\ Email: makoto.takizawa@computer.org
}

\begin{abstract}
A set of wireless mobile terminals, which cooperate by routing packets to each other create a Mobile Ad hoc Network (MANET). MANETs are attracting attention for their potential use in several fields such as collaborative computing and disaster recovery environments. Considering mobility of the terminals, the routes change dynamically and routing becomes a key process for operation of MANETs. Until now we considered only the Constant Bit Rate (CBR) transmission over UDP transport protocol in our testbed. In this paper, we analyse the performance of our MANET testbed in an indoor environment considering multimedia transmissions with variable packet rate. Using linear and mesh topologies for connecting the nodes, and BATMAN routing protocol, we measure throughput, delay and packet loss metrics.
\end{abstract}

Keywords-Multimedia Transmissions; MANET Testbed; Problems and Issues; BATMAN; Audio; Video

\section{INTRODUCTION}

A collection of wireless mobile hosts that can dynamically establish a temporary network without any aid from fixed infrastructure is known as a Mobile Ad-hoc Network (MANET). The mobile hosts act as routers for each other and they are connected via wireless links. Recently, MANETs are continuing to attract the attention for their applications in several fields, where the communication infrastructure is expensive and/or time consuming. Mobility and the absence of any fixed infrastructure make MANET very attractive for rescue operations and timecritical applications.

Research for MANETs has been done usually in simulation, because in general, a simulator can give a quick and inexpensive evaluation of protocols and algorithms.
However, experimentations in the real world [1] are very important to verify the simulation results and to revise the models implemented in the simulator. A typical example of this approach has revealed many aspects of IEEE 802.11 , like the gray-zones effect [2], which usually are not taken into account in standard simulators, as the wellknown $n s-2$ simulator.

We conducted many experiments with our MANET testbed, which are shown in our previous works [3], [4]. We proved that while some of the Optimized Link State Routing (OLSR) problems can be solved (for instance the routing loop), but this protocol still has the self-interference problem. There is an intricate interdependence between MAC layer and routing layer, which can lead the experimenter to misunderstand the results of the experiments. For example, the horizon is not caused only by IEEE 802.11 Distributed Coordination Function (DCF), but also by the routing protocol.

We carried out the experiments with different routing protocols such as OLSR and Better Approach to Mobile Ad-hoc Networks (BATMAN) and found that throughput of TCP was improved by reducing Link Quality Window Size (LQWS), but there were packet loss because of experimental environment and traffic interference. For TCP data flow, we got better results when the LQWS value was 10. Moreover, we found that the node join and leave operations affect more the TCP throughput and Round Trip Time (RTT) than UDP [5]. In [6], we showed that BATMAN buffering feature showed a better performance than Ad-hoc On-demand Distance Vector (AODV), by handling the communication better when routes changed 
dynamically.

Many researchers performed valuable research in the area of wireless multi-hop networks by computer simulations and experiments [7], [8]. Most of them are focused on throughput improvement, and they do not consider mobility [9]. In [10], [11], the authors propose a dynamic probabilistic broadcasting scheme for mobile ad-hoc networks where nodes move according to different mobility models. Simulation results show that their approach outperforms the Fixed Probability Ad hoc On-demand Distance Vector (FP-AODV) and simple AODV in terms of saved rebroadcast under different mobility models. It also achieves higher saved rebroadcast and low collision as well as low number of relays than the fixed probabilistic scheme and simple AODV.

In [12], the authors implemented multi-hop mesh network called Massachusetts Institute of Technology (MIT) Roofnet, which consists of about 50 nodes. They consider the impact of node density and connectivity in the network performance. The authors show that the multi-hop link is better than single-hop link in terms of throughput and connectivity. In [13], the authors analyse the performance of an outdoor ad-hoc network, but their study is limited to reactive protocols such as AODV [14] and Dynamic Source Routing (DSR) [15].

In [16], the authors perform outdoor experiments of non standard proactive protocols. Other ad-hoc experiments are limited to identify MAC problems, by providing insights on the one-hop MAC dynamics as shown in [17]. In [18], the disadvantage of using hysteresis routing metric is presented through simulation and indoor measurements.

In [19], the authors presents performance of OLSR using the standard hysteresis routing metric and the Expected Transmission Count (ETX) metric in a 7 by 7 grid of closely spaced Wi-Fi nodes to obtain more realistic results. The throughput results are effected by hop distance, similar to our previous work [3].

The authors of [20] described the performance of the BATMAN protocol on an indoor testbed, using Mesh Potato (MP) devices. The MPs are small devices used for voice communications over the wireless medium but also support data. The Batman-adv protocol is designed for ad hoc wireless networks. They use variable packet size to evaluate the performance.

In this work, we analyse the performance of BATMAN [21] routing protocol in an indoor environment, using linear and mesh topologies. We designed a MANET testbed, consisting of five mobile machines. The data sent through the network are of two different types, resembling to audio and video transmissions. We investigate the performance of the system by measuring throughput, delay and packet loss.

The structure of the paper is as follows. In Section II, we give an overview of BATMAN routing protocol. In Section III, we describe the design and implementation of the MANET testbed. In Section IV, we discuss the experimental results and evaluate the performance of the testbed. Finally, we draw conclusions in Section V.

\section{Overview OF BATMAN Routing Protocol}

In OLSR, there is a serious synchronization problem between the topology messages and the routing information stored inside every node. In other words, a mismatch between what is currently stored in the routing tables and the actual topology of the network may arise. This is due to the propagation time of the topology messages. Routing loops are the main effect of such problem. To solve this problem, BATMAN has been introduced. In BATMAN, there is no topology message dissemination. Every node executes the following operations.

1) Sending of periodic advertisement messages, called OriGinator Message (OGM). The size of these messages is just 52 bytes, containing: the IP address of the originator, the IP address of the forwarding node, a Time To Live (TTL) value and an increasing Sequence Number (SQ).

2) Checking of the best one-hop neighbor for every destination in the network by means of a ranking procedure.

3) Re-broadcasting of OGMs received via best one-hop neighbour.

The BATMAN uses a timer for sending OGMs and SQ (OGM) for checking the bi-directionality of links. If the SQ of an OGM received from a particular node falls within a certain range, the corresponding link is considered bidirectional. For example, suppose that in a time interval $T$, the node A sends $T r$ messages, where $r$ is the rate of OGM messages. The neighbours of A will re-broadcast the OGMs of A and also other node's OGMs. When A receives some OGMs from a neighbour node, say $B$, it checks if last received OGM from B has a SQ less or equal to $T r$. If it does, then $\mathrm{B}$ is considered bi-directional, otherwise it is considered unidirectional. Bi-directional links are used for the ranking procedure. The quantity $\mathrm{Tr}$ is called bi-directional sequence number range. The ranking procedure is a substitute of the link quality extension of OLSR. In few words, every node ranks its neighbouring nodes by means of a simple counting of total received OGMs from them. The ranking procedure is performed on OriGinator (OG) basis, i.e. for every originator. Initially, for every OG, every node stores a variable called Neighbour Ranking Sequence Frame (NBRF), which is upper bounded by a particular value called ranking sequence number range. We suppose that there is a rank table in every node which stores all the information contained in the OGMs. Whenever a new OGM is being received via a bi-directional link, the receiving node executes the following steps.

1) If the sequence number of the OGM is less than the corresponding NBRF, then drop the packet.

2) Otherwise, update the $\mathrm{NBRF}=\mathrm{SQ}(\mathrm{OGM})$ in the ranking table.

3) If $\mathrm{SQ}(\mathrm{OGM})$ is received for the first time, store OGM in a new row of the rank table.

4) Otherwise, increment by one the OGM count or make ranking for this OGM. 


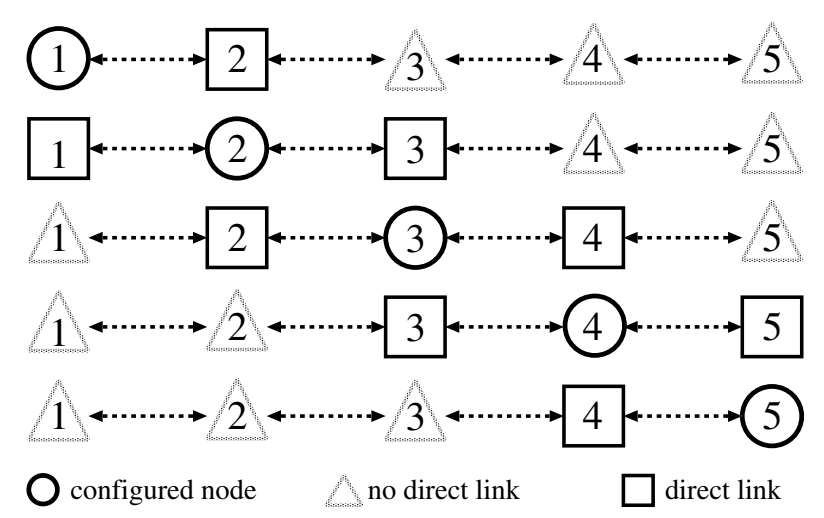

(a) Node 1

Figure 1. Node positioning and MAC filtering for LT.

Finally, the ranking procedures select the best onehop neighbour, the neighbour which has the highest rank in the ranking table. Let us note that the same OGM packet is used for: link sensing, neighbour discovery, bidirectional link validation and flooding mechanism. While this feature eliminates routing loops because no global topology information are flooded, the self-interference due to data traffic can cause oscillations in the throughput. Other details on BATMAN can be found in [21].

In BATMAN, every node re-broadcasts received OGMs only once (only those OGMs which have been received via the best-ranked neighbour). This is a kind of selective flooding, which practically reduces the overhead of the flooding. Another analogy can be found in gossip protocols [22]. In gossip protocol, every node decides to re-broadcast received data with some probability, $p$. This is equivalent with eliminating some links in the network and then supposing that every node re-broadcast with probability 1 . In gossip protocol there is a threshold for $p$ and the density of nodes after which the success ratio ${ }^{1}$ is almost surely 1 . In BATMAN, the probability $p$ is changed according to ranking procedure. It is the probability that an OGM is reached via the best rank neighbour. Let us note that the selective flooding eliminates possible misbehaviour of the ranking procedure. In fact, cumulative count of the OGM could be greater than the total number of OGM received via the current best neighbour.

\section{DESIGN AND IMPLEMENTATION OF MANET TESTBED}

\section{A. Testbed Description}

The machines operate on Fedora 14 OS with kernel 2.6.35 suitably modified in order to support the wireless cards. The lynksys wireless network cards (Model: WUSB54G ver. 4) are usb-based cards with an external antenna of $2 \mathrm{dBi}$ gain, transmitting power of $16+/-1 \mathrm{dBm}$ and receiving sensitivity of $-80 \mathrm{dBm}$. We verified that the external antenna improves the quality of the first hop link,

\footnotetext{
${ }^{1}$ That is the probability that broadcast messages are received by all nodes in the network.
}

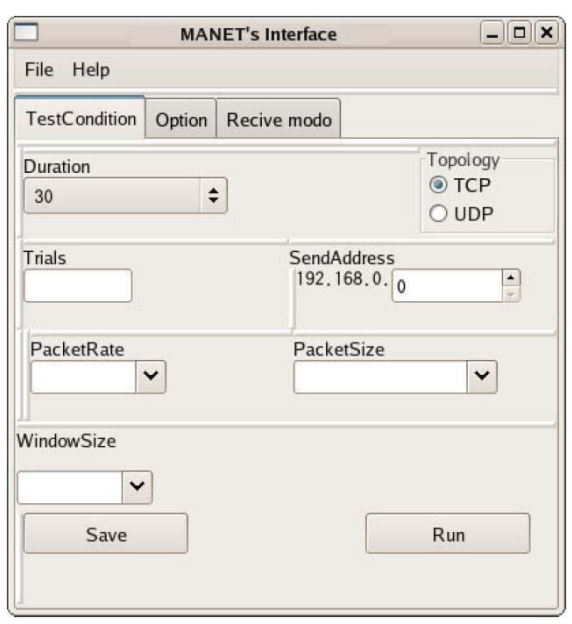

Figure 3. Testbed interface.

which is the link connecting the ad-hoc network. The driver can be downloaded from the web site [23], [24]. We show screenshots of these machines in Fig. 2.

In our testbed, we have two systematic traffic sources we could not eliminate: the control traffic and the other wireless Access Points (APs) interspersed within the campus. The control traffic is due to the ssh program, which is used to remotely start and control the measurement software on the source node. The other traffic source brings interferences occupying the available bandwidth, which is typical in an academic scenario.

The traffic in the network is sent by Distributed Internet Traffic Generator (D-ITG) software, version 2.7.0 Beta2, which is an Open Source Traffic Generator [25]. With DITG, we can inject different type of data flows in the network. After finishing the transmission, D-ITG offers decoding tools to get information about different metrics along the whole transmission duration.

\section{B. Testbed Interface}

Upon the first implementation of the testbed, all the parameters settings and editing were done by using command lines of bash shell (terminal), which resulted in many misprints and the experiments were repeated many times. In order to make the experiments easier, we implemented a Graphical User Interface (GUI). We used wxWidgets tool and each operation is implemented by Perl language. wxWidgets is a cross-platform GUI and tools library for GTK, MS Windows and Mac OS.

We implemented many parameters in the interface such as transmission duration, number of trials, source address, destination address, packet rate, packet size and topology setting function. We can save the data for these parameters in a text file and can manage in a better way the experimental conditions. Moreover, we implemented collection function of experimental data in order to make easier the experimenter's work. A screenshot of the interface is shown in Fig. 3. 


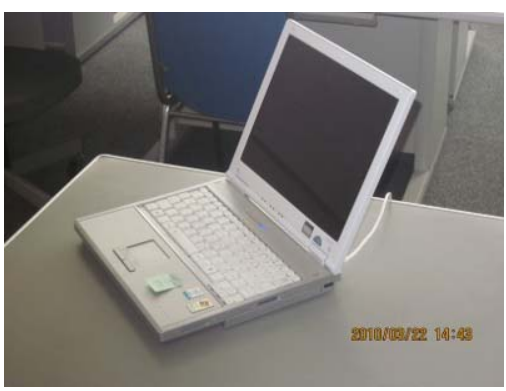

(a) Node 1

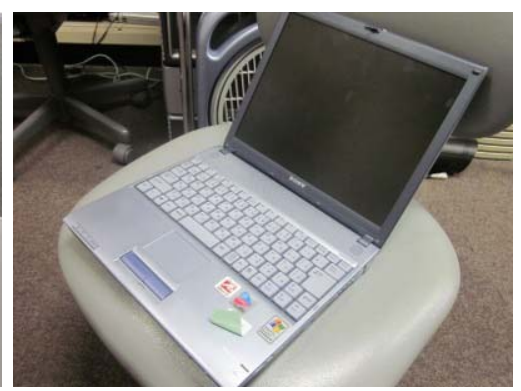

(b) Node 2

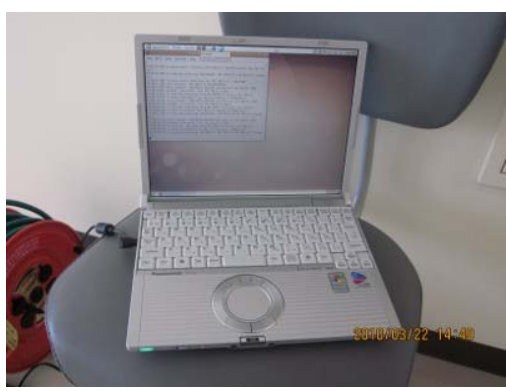

(c) Node 3

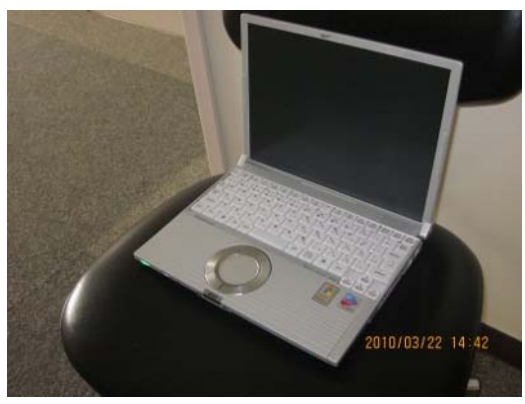

(d) Node 4

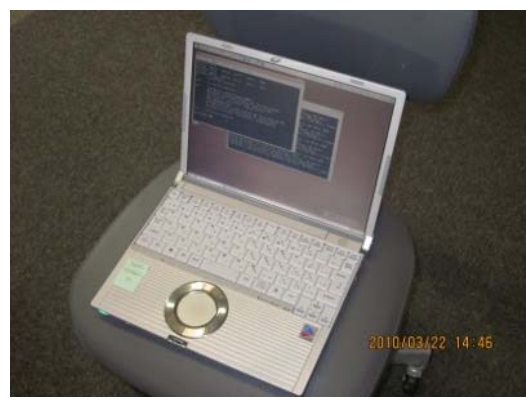

(e) Node 5

Figure 2. Screenshots of each node.

\section{Experimental Environment}

Our testbed provides an experimental platform for evaluating protocols and algorithms using realistic parameters. In this testbed, we can implement different topology scenarios and analyse different routing protocols considering different metrics. In this work, we take the following considerations.

- The experiments are conducted in an indoor environment, inside the fifth floor of our departmental building.

- We analyse our network for two experimental topologies: Linear Topology (LT) and Mesh Topology (MT).

- The packet's size is 60 bytes.

- We sent data with different packet rates, resembling audio data (20-50 pps) and video data (100-800 pps). From now on, we will refer to Audio and Video, respectively.

- We discuss the effect of multi-hop and data size regarding throughput, delay and packet loss using BATMAN routing protocol.

\section{EXPERIMENTAL RESULTS}

\section{A. Experimental Settings}

The experimental parameters are shown in Table I. We study the impact of best-effort traffic for LT and MT. For LT, we enabled MAC filtering routines, using iptables. We collected data for throughput, delay and packet loss, using D-ITG.

The data sent to conduct the experiments is transported over UDP. In our previous works [4]-[6], we used a fixed packet rate for sending the CBR flow over the network.
Table I

EXPERIMENTAL PARAMETERS.

\begin{tabular}{|l|c|}
\hline Parameters & Values \\
\hline \hline Number of Nodes & 5 \\
MAC & IEEE $802.11 \mathrm{~b} /$ Channel 2 \\
Transmitted Power & $16+/-1 \mathrm{dBm}$ \\
OS (kernel) & Fedora 14 (2.6.35) \\
Traffic Generator & D-ITG 2.7.0 Beta2 \\
Packet Rate & $20-50,100-800 \mathrm{pps}$ \\
Packet Size & 60 bytes \\
Number of Trials & 10 \\
Duration & $120 \mathrm{sec}$ \\
Routing Protocol & BATMAN \\
\hline
\end{tabular}

We believe that by making the packet rate variable, the bandwidth can be used more efficiently. For the Audio data we send the packets with a uniformly distributed rate, varying from 20 to $50 \mathrm{pps}$. The throughput for this data will vary from $9.6 k b p s$ to $24 k b p s$, which is almost the same as the throughput for GSM or UMTS audio communications, [26]. For the Video data, we use a uniformly distributed packet rate, from 100 to $800 \mathrm{pps}$, which corresponds to 48 and $384 \mathrm{kbps}$, respectively.

Throughput, delay and packet loss of UDP traffic are computed at the receiver. We show the average data of these metrics in tables and we display the measured throughput plotted in time domain, in order to see the oscillations during the experiment.

As MAC protocol, we used IEEE 802.11b. The transmission power was set in order to guarantee a radius covering at least one hop physical distance. Since we 


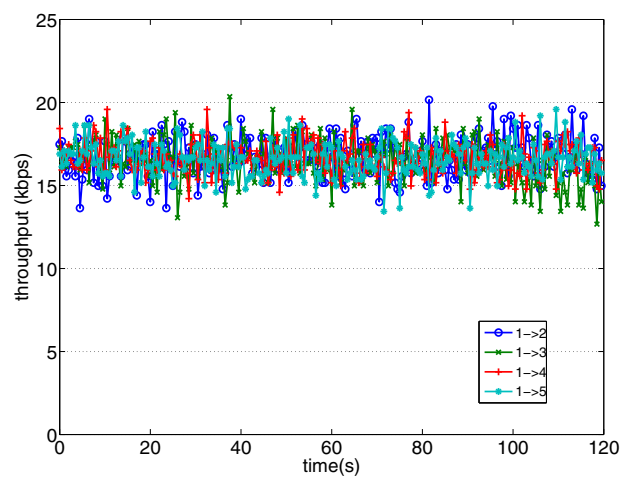

(a) Audio data, LT

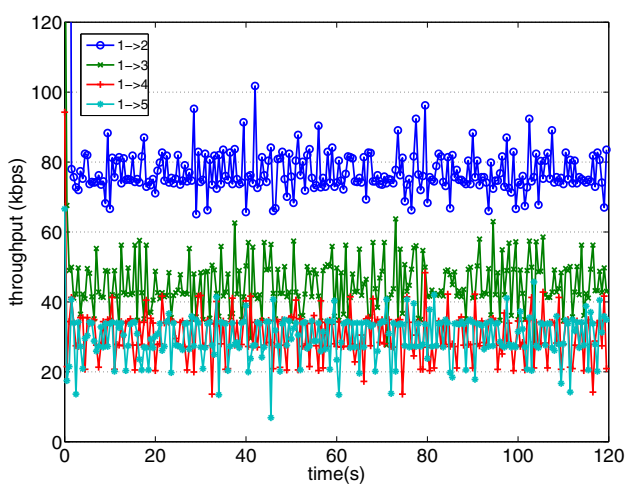

(c) Video data, LT

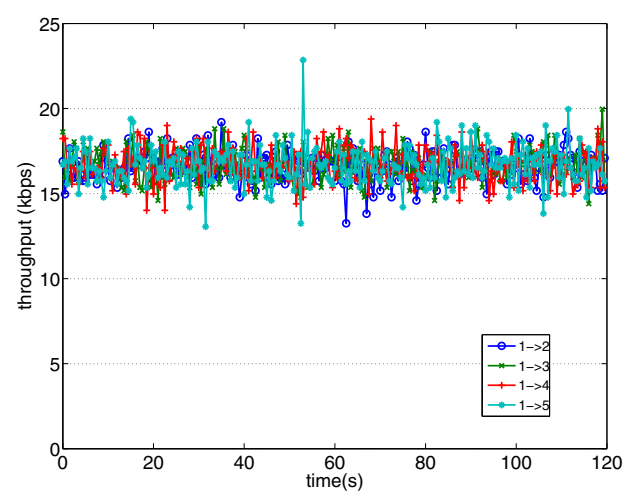

(b) Audio data, MT

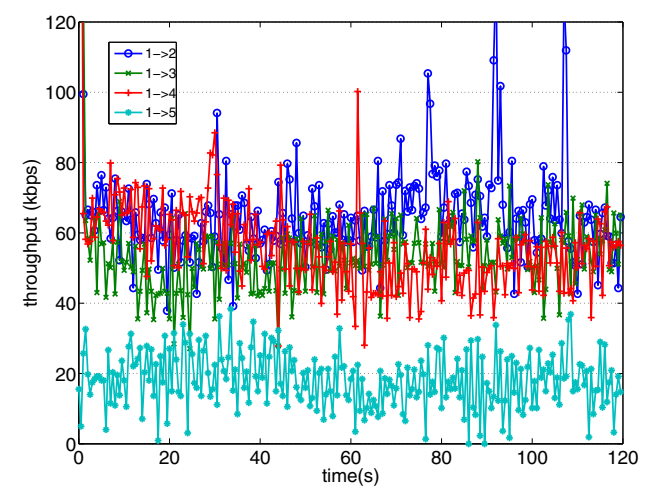

(d) Video data, MT

Figure 4. Throughput results.

Table II

THROUGHPUT RESULTS (KBIT/S).

\begin{tabular}{|c|c|c|c|c|c|c|c|c|c|c|c|c|}
\hline \multirow{3}{*}{ Flow } & \multicolumn{6}{|c|}{ Audio (9.6-24 kbps) } & \multicolumn{6}{|c|}{ Video (48-384 kbps) } \\
\hline & \multicolumn{3}{|c|}{ LT } & \multicolumn{3}{|c|}{ MT } & \multicolumn{3}{|c|}{ LT } & \multicolumn{3}{|c|}{ MT } \\
\hline & $\min$ & $\max$ & $\mathrm{av}$ & $\min$ & $\max$ & av & $\min$ & $\max$ & av & $\min$ & $\max$ & av \\
\hline$\overline{\# \# 1 \rightarrow \# 2}$ & 13.63 & 20.16 & 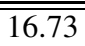 & 13.25 & 19.20 & 16.57 & 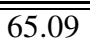 & $\overline{360.19}$ & 80.12 & 38.82 & $\overline{220.99}$ & 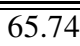 \\
\hline$\# 1 \rightarrow \# 3$ & 12.67 & 20.35 & 16.46 & 14.40 & 19.97 & 16.69 & 27.65 & 161.86 & 45.41 & 27.07 & 253.06 & 55.04 \\
\hline$\# 1 \rightarrow \# 4$ & 14.21 & 19.58 & 16.64 & 14.02 & 19.39 & 16.69 & 13.63 & 94.27 & 30.74 & 27.84 & 226.18 & 56.87 \\
\hline$\# 1 \rightarrow \# 5$ & 13.44 & 19.58 & 16.58 & 13.06 & 22.85 & 16.67 & 6.91 & 66.24 & 30.08 & 0.96 & 38.40 & 18.28 \\
\hline
\end{tabular}

Table III

DELAY RESUlTS (S)

\begin{tabular}{|c|c|c|c|c|c|c|c|c|c|c|c|c|}
\hline \multirow{3}{*}{ Flow } & \multicolumn{6}{|c|}{ Audio (9.6-24 kbps) } & \multicolumn{6}{|c|}{ Video $(48-384 \mathrm{kbps})$} \\
\hline & \multicolumn{3}{|c|}{ LT } & \multicolumn{3}{|c|}{ MT } & \multicolumn{3}{|c|}{ LT } & \multicolumn{3}{|c|}{ MT } \\
\hline & $\min$ & $\max$ & av & $\min$ & $\max$ & av & $\min$ & $\max$ & av & $\min$ & $\max$ & av \\
\hline $\bar{~} \# 1 \rightarrow \# 2$ & $\overline{0.006}$ & 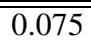 & $\overline{0.012}$ & 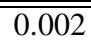 & 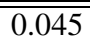 & 0.004 & $\overline{0.081}$ & 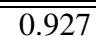 & 0.735 & 0.150 & $\bar{~} 1.640$ & 1.285 \\
\hline$\# 1 \rightarrow \# 3$ & 0.007 & 0.063 & 0.015 & 0.002 & 0.066 & 0.005 & 0.181 & 3.978 & 3.581 & 0.121 & 1.801 & 1.137 \\
\hline$\# 1 \rightarrow \# 4$ & 0.005 & 0.092 & 0.016 & 0.002 & 0.086 & 0.004 & 0.230 & 6.742 & 5.161 & 0.155 & 2.581 & 1.497 \\
\hline$\# 1 \rightarrow \# 5$ & 0.007 & 0.117 & 0.018 & 0.002 & 0.088 & 0.007 & 0.236 & 12.955 & 9.461 & 0.221 & 14.154 & 6.010 \\
\hline
\end{tabular}

were interested mainly in the performance of the routing protocol, we kept unchanged all MAC parameters, such as the carrier sense, the retransmission counter, the con- tention window and the RTS/CTS threshold. Moreover, the channel central frequency was set to $2.417 \mathrm{GHz}$ (channel 2). In regard to the interference, it is worth noting that, 
Table IV

PACKET LOSS RESULTS (PPS).

\begin{tabular}{|c|c|c|c|c|c|c|c|c|c|c|c|c|}
\hline \multirow{3}{*}{ Flow } & \multicolumn{6}{|c|}{ Audio (9.6-24 kbps) } & \multicolumn{6}{|c|}{ Video (48-384 kbps) } \\
\hline & \multicolumn{3}{|c|}{ LT } & \multicolumn{3}{|c|}{ MT } & \multicolumn{3}{|c|}{ LT } & \multicolumn{3}{|c|}{ MT } \\
\hline & $\min$ & $\max$ & av & $\min$ & $\max$ & av & $\min$ & $\max$ & av & $\min$ & $\max$ & av \\
\hline$\overline{\# 1 \rightarrow \# 2}$ & 0 & 0.2 & $\overline{0.003}$ & 0 & 0.2 & 0.004 & $\overline{0}$ & 372.6 & 128.8 & 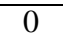 & $\overline{66042}$ & 237.5 \\
\hline$\# 1 \rightarrow \# 3$ & 0 & 1.2 & 0.026 & 0 & 0.4 & 0.005 & 0 & 364.0 & 138.8 & 0 & 7021 & 256.8 \\
\hline$\# 1 \rightarrow \# 4$ & 0 & 1.4 & 0.043 & 0 & 0.2 & 0.003 & 0 & 326.8 & 145.8 & 0 & 7408 & 283.1 \\
\hline$\# 1 \rightarrow \# 5$ & 0 & 2.0 & 0.032 & 0 & 2.6 & 0.025 & 0 & 395.2 & 171.7 & 0 & 8620 & 405.2 \\
\hline
\end{tabular}

during our tests, almost all the IEEE 802.11 spectrum had been used by other APs disseminated within the campus.

\section{B. Experimental Analysis}

We show throughput results during 120 seconds of data transmission, in Fig. 4. In Tables. II, III and IV, are shown the minimum, maximum and average values of throughput, delay and packet loss, respectively. In Figs. 4(a) and 4(b), is shown the throughput of Audio flow transmission for both LT and MT. We notice that the performance is almost the same for both topologies. The throughput has a few oscillations, but it stays at almost the same levels for all communications $(1 \rightarrow 2,1 \rightarrow 3,1 \rightarrow 4$ and $1 \rightarrow 5)$. If we look at Fig. 4(c), where we have a Video transmission with LT, the performance for $1 \rightarrow 2$ flow is the best, and the performance decrease as the number of hops increases up to 4 hops in $1 \rightarrow 5$ flow. However 2-hop and 3-hop communications have similar performance. On the other hand, for MT in Fig. 4(d), we can see that the performance for $1 \rightarrow 2,1 \rightarrow 3$ and $1 \rightarrow 4$ flows are almost similar, while in case of $1 \rightarrow 5$ flow, the values of throughput fall to around $20 \mathrm{kbps}$.

Comparing the results of Fig. 4 and Table II, we can see clearly that for Audio (small data rates) there is no difference between LT and MT, but for larger data rates, in MT case the average throughput decreases and its oscillations increase. In LT scheme, one node can receive OGMs only from its neighbours, so the number of received OGMs from other nodes is zero. This means that the route from node 1 to other nodes, are always known and unchangeable. However there is an unchangeable 4-hop transmission from node 1 to node 5. In the MT scheme, lets say, node 1 wants to transmit to node 5 with the high rate Video transmission of 48-384 pps. First, the best 1hop neighbour is node 2 and it starts to send packets to node 2 . Meanwhile, the $1 \rightarrow 2$ link is busy and node 1 receives from node 3 some OGMs broadcast by node 5 , so that node 3 becomes the best 1-hop neighbour. Then, while the $1 \rightarrow 3$ link is busy, it will happen the opposite. This instability in routes, brings oscillations and decrease in throughput. The packet loss, shown in Table IV, is closely related to throughput and it has a similar behaviour. We can see that for Audio data the packet loss is very small so the performance is very good.

Regarding delay, we show the results in Table III. The average delay for Audio transmissions, for LT and
MT is under $20 \mathrm{~ms}$ and $10 \mathrm{~ms}$, respectively. For video transmissions, delay reaches values up to 9 seconds, in case of $1 \rightarrow 5$ flow for LT. The number of hops for LT is 4 hops. Packets travelling through 4 hops, takes more time to reach the destination.

\section{Conclusions}

In this work, we designed and implemented two topologies in our MANET testbed in indoor environment. We used LT and MT to evaluate the performance of BATMAN protocol, in cases of low data rates (Audio) and high data rates (Video). We investigated throughput, delay and packetloss metrics. From our analysis we found the following results.

- For Audio transmissions, the performance was almost the same for both LT and MT topologies. The throughput had a few oscillations.

- The average delay values were under $20 \mathrm{~ms}$ and 10 $\mathrm{ms}$, for LT and MT, respectively.

- Packet loss is less than 1 pps for all cases of Audio data.

- For Video transmissions with higher data rates, the performance decreased as the number of hops increases up to 4 hops in $1 \rightarrow 5$ flow.

- For MT, we could see that the performance for $1 \rightarrow 2$, $1 \rightarrow 3$ and $1 \rightarrow 4$ flows were almost similar, while in case of $1 \rightarrow 5$ flow, the values of throughput fell to around 20kbps.

- We could see that for Audio data there is no difference between LT and MT, but for Video data, in MT case the average value of throughput decreased and its oscillations increased.

- The packet loss, is closely related to throughput so it had a similar behaviour.

- In LT scheme, routes are static. While in MT scheme, routes change and this decreased throughput and increased packet loss.

- Delay values were higher in the case of LT scheme because of more number of hops.

In this experiments, all nodes were static. We would like to make the nodes dynamic and investigate the performance of BATMAN protocol in different conditions. We also plan to study the behaviour of ranking procedure of BATMAN. Moreover, we would like to compare experimental results with simulation data. 


\section{ACKNOWLEDGEMENT}

This work is supported by a Grant-in-Aid for scientific research of Japan Society for the Promotion of Science (JSPS). The authors would like to thank JSPS for the financial support.

\section{REFERENCES}

[1] W. Kiess and M. Mauve, "A Survey on Real-world Implementations of Mobile Ad-hoc Networks", Journal of AdHoc Networks, Vol. 5, No. 3, pp. 324-339, 2007

[2] H. Lundgren, E. Nordstrom and C. Tschudin, "Coping with Communication Gray Zones in IEEE 802.11b Based AdHoc Networks", Proc. of International Workshop on Wireless Mobile Multimedia (WOWMOM-2002/MOBICOM2002), pp. 49-55, 2002.

[3] G. De Marco, M. Ikeda, T. Yang and L. Barolli, "Experimental Performance Evaluation of a Pro-active Adhoc Routing Protocol in Outdoor and Indoor Scenarios", Proc. of International Conference on Advanced Information Networking and Applications (AINA-2007), pp. 7-14, May 2007.

[4] L. Barolli, M. Ikeda, G. De Marco, A. Durresi and F. Xhafa, "Performance Analysis of OLSR and BATMAN Protocols Considering Link Quality Parameter", Proc. of International Conference on Advanced Information Networking and Applications (AINA-2009), pp. 307-314, May 2009

[5] M. Ikeda, L. Barolli, M. Hiyama, T. Yang, G. De Marco and A. Durresi, "Performance Evaluation of a MANET Testbed for Different Topologies," Proc. of International Conference on Network-Based Information Systems (NBiS-2009), Indianapolis, pp. 327-334, August 2009.

[6] E. Kulla, M. Ikeda, L. Barolli and R. Miho, "Impact of Source and Destination Movement on MANET Performance Considering BATMAN and AODV Protocols", Proc. of International Conference on Broadband, Wireless Computing, Communication and Applications (BWCCA2010), pp. 94-101, 2010

[7] E. Nordström, "APE - A Large Scale Ad-hoc Network Testbed for Reproducible Performance Tests", Masters thesis, Uppsala University, 2002.

[8] Y. Owada, Y. Takahashi, T. Suda, H. Terui, F. Taki, T. Yagi, and K. Mase, "A Large Scale Wireless Mobile Adhoc Network Testbed", Proc. of IEEE Vehicular Technology Conference, pp. 324-328, September 2005.

[9] R. Draves, J. Padhye, and B. Zill, "Comparison of Routing Metrics for Static Multi-hop Wireless Networks", Proc. of International Conference on Special Interest Group on Data Communication (SIGCOMM-2004), pp. 133-144, 2004.

[10] A. M. Hanashi, A. Siddique, I. Awan, and M. Woodward, "Performance Evaluation of Dynamic Probabilistic Flooding Under Different Mobility Models in MANETs", Proc. of International Conference on Parallel and Distributed Systems (ICPADS-2007), Vol. 2, pp. 1-6, December 2007.

[11] A. M. Hanashi, I. Awan, and M. Woodward, "Performance Evaluation with Different Mobility Models for Dynamic Probabilistic Flooding in MANETs", Journal on Mobile Information Systems (IJMIS), Vol. 5, No. 1, pp. 65-80, 2009.
[12] J. Bicket, D. Aguayo, S. Biswas, and R. Morris, "Architecture and Evaluation of an Unplanned 802.11b Mesh Network", Proc. of International Conference on Mobile Computing and Networking (MOBICOM-2005), pp. 31-42 2005

[13] D. A. Maltz, J. Broch, and D. B. Johnson, "Lessons from a Full-scale Multihop Wireless Ad-Hoc Network Testbed", Journal on Personal Communications, Vol. 8, No. 1, pp. 8-15, February 2001.

[14] C. Perkins, E. Belding-Royer, and S. Das, "Ad-hoc OnDemand Distance Vector (AODV) Routing", RFC 3561 (Experimental), Jul. 2003.

[15] D. B. Johnson, D. A. Maltz, and J. Broch, "DSR: The Dynamic Source Routing Protocol for Multi-Hop Wireless Ad-Hoc Networks", Journal on Ad-Hoc Networking, Chap. 5, pp. 139-172, 2001.

[16] R. S. Gray, D. Kotz, C. Newport, N. Dubrovsky, A. Fiske, J. Liu, C. Masone, S. McGrath and Y. Yuan, "Outdoor Experimental Comparison of Four Ad-Hoc Routing Algorithms", Proc. of Modelling, Analysis and Simulation of Wireless Mobile Systems (MSWiM-2004), pp. 220-229, 2004.

[17] E. Borgia, M. Conti, and E. Gregori, "IEEE 802.11b AdHoc Networks: Performance Measurements", Journal of Cluster Computing, Vol. 8, No. 2-3, pp. 135-145, 2005.

[18] T. Clausen, G. Hansen, L. Christensen, and G. Behrmann, "The Optimized Link State Routing Protocol, Evaluation through Experiments and Simulation", Proc. of IEEE Symposium on Wireless Personal Mobile Communications, Available online at http://hipercom.inria.fr/olsr/wpmc01.ps, September 2001

[19] D. Johnson and G. Hancke, "Comparison of Two Routing Metrics in OLSR on a Grid based Mesh Network", Journal on Ad-Hoc Networks, Vol. 7, No. 2, pp. 374-387, March 2009 .

[20] E. Chissungo, E. Blake, H. Le, "Investigation into Batmanadv Protocol Performance in an Indoor Mesh Potato Testbed", Proc. of IEEE International Conference on Intelligent Networking and Collaborative Systems (IEEE INCoS2011), pp. 8-13, 2011

[21] Open Mesh Webpage for a Collection of Tools to Build Free and Open Mesh Networks, Available online at http://www.open-mesh.org/

[22] Z.J. Haas, J.Y. Halpern and L. Li, "Gossip-based ad-hoc routing", IEEE/ACM Transactions on Networking, Vol. 14, No. 3, pp. 479-491, 2006.

[23] Rt2x00 Project for Linux Wireless Cards Drivers, Available online at: http://rt2x00.serialmonkey.com/.

[24] Ralink RT2570 USB Enhanced Driver, Available online at: http://homepages.tu-darmstadt.de/p_larbig/wlan/.

[25] A. Dainotti, A. Botta, and A. Pescap, "Do you know what you are generating?" Proc. of International Conference on Emerging Networking Experiments and Technologies (CoNEXT-2007), New York, USA, pp. 1-2, 2007.

[26] F. Hillebrand, "GSM and UMTS: The Creation of Global Mobile Communication”, Chichester [u.a.] Wiley, 2002. 